\title{
Corticosteroids: Use or Misuse in Veterinary Medicine
}

\author{
Asmita Narang* and Gurpreet Singh Preet \\ Department of Veterinary Medicine, Guru Angad Dev Veterinary and Animal Sciences \\ University, Ludhiana, India \\ *Corresponding author
}

\section{A B S T R A C T}

Due to broad range of effects, corticosteroids are frequently used in

Keywords

Corticosteroids,

Glucocorticoids,

Antiinflamatory,

Immunosuppression

Article Info

Accepted:

10 July 2019

Available Online:

10 August 2019

veterinary practice. Wide ranges of biological effects are mediated via different dose and potency of different glucocorticoids. Intensive short-term glucocorticoid use can decrease the morbidity and mortality of potentially fatal conditions but in long run their use can lead to immunosuppression, duodenal ulceration, pancreatitis, adrenal atrophy, hyperadrenocorticism, glucose intolerance and many more diseases. Multitude of adverse effects distinct from pharmacologic or physiologic responses has also been associated with corticosteroid use. The dose should be tapered and changed to alternate-day therapy as soon as possible, but caution should be taken not to allow recurrence of disease. The underlying disease must also be directly addressed as corticosteroids most often only suppress symptoms without treating the disease.

\section{Introduction}

The adrenal cortex synthesizes two classes of steroids: the corticosteroids (glucocorticoids and mineralocorticoids), have 21 carbon atoms and the androgens, which have 19. The actions of corticosteroids historically were described as glucocorticoid (carbohydrate metabolism-regulating) and mineralocorticoid (electrolyte balance-regulating), reflecting their preferential activities (Goodman, 1996).

The glucocorticoids (such as cortisol and related synthetics, prednisone and dexamethasone and numerous others) act on the mechanics of carbohydrates, fat, and protein metabolism and mineralocorticoids (such as aldosterone and related synthetic fludrocortisone acetate) influence the electrolytes: sodium and potassium.

Although necessary for maintenance of homeostasis, these powerful drugs produce significant side effects when given in pharmacological dosages. Their uses are broad ranging, but are mainly used for their antiinflammatory and immunosuppressive effects (Torres et al., 2005). 


\section{Physiology and mechanism of action}

Corticosteroids are primarily produced from zona glomerulosa and zona fasciculata of the adrenal gland and have a plethora of functions (Behrend and Greco, 1997). Corticosteroids are constantly synthesized under the control of the hypothalamus and pituitary. Cortisol and corticosterone concentrations in plasma influence ACTH secretion (Boothe and Mealey, 2001). Exogenous corticosteroid administration can also suppress ACTH secretion, with the degree of suppression depending on the particular drug administered (MacDonald, 2000).

Steroids are thought to enter the cell by passive diffusion. At least three steroid receptors have been identified and associated with different physiologic effects (Funder, 1992). Every cell type has glucocorticosteroid receptors (GR), with the type and concentration of the particular receptor varying between species and tissue (Boothe and Mealey, 2001).

\section{Physiological effects of glucocorticoids}

The effects of corticosteroids are numerous and widespread, and include alterations in carbohydrate, protein, and lipid metabolism; maintenance of fluid and electrolyte balance; and preservation of normal function of the cardiovascular system, the immune system, the kidney, skeletal muscle, the endocrine system, and the nervous system (Standora et al., 2017). GCs stimulate protein catabolism, which results in increased serum concentrations of amino acids. The metabolism of lipids is also affected by glucocorticosteroids, which promote lipolysis and inhibit long-chain fatty acid synthesis (MacDonald, 2000). Glucocorticoids have a permissive effect on a number of other metabolic reactions. Glucocorticoids influence water and electrolyte balance through mineralocorticoid actions. Synthetic glucocorticoids possess varying degrees of mineralocorticoid activity, but all have less than $1 \%$ of the mineralocorticoid activity of aldosterone. Glucocorticoids have an inhibitory effect on antidiuretic hormone and may decrease the permeability of the distal renal tubules to water via a direct action (MacDonald, 2000). The actions of corticosteroids are interrelated to those of other hormones. For example, in the absence of lipolytic hormones, cortisol has virtually no effect on the rate of lipolysis by adipocytes. Likewise, in the absence of glucocorticoids, epinephrine and norepinephrine have only minor effects on lipolysis. Administration of a small dose of glucocorticoid, however, markedly potentiates the lipolytic action of these catecholamines (Sapolsky, 2000). Glucocorticoids exert minor effects on hemoglobin and erythrocyte content of in Cushing's syndrome. More profound effects are seen in the setting of autoimmune hemolytic anemia, in which the immunosuppressive effects of glucocorticoids can diminish the self-destruction of erythrocytes (Sapolsky, 2000).

Glucocorticoids exert their action on leukocyte numbers as well as function, ultimately impacting both humoral and cellmediated arms of the immune response (Behrend and Greco, 1997). The immunosuppressive effects of glucocorticosteroids are more pronounced on the cellular arm than on the humoral arm of the immune system (MacDonald, 2000). The immunosuppressive actions of glucocorticosteroids, like their antiinflammatory actions, involve disruption of the intercellular communication of leukocytes via interference with lymphokine production, biologic action or both (Bone, 1991). Glucocorticoids indirectly affect the central nervous system by maintaining normal blood glucose concentrations and maintaining 
normal fluid and electrolyte concentrations (Sapolsky, 2000). In humans, glucocorticosteroids are believed to influence mood (including euphoria), behavior, and brain excitability. The euphoric effect commonly recognized in dogs is likely to reflect differences in glucocorticosteroid receptors (Boothe and Mealey, 2001).

\section{Pharmacological effects of glucocorticoids}

Glucocorticoids vary with respect to potency, mineralocorticoid effect, antiinflammatory effect, onset of action, plasma half-life and duration of biological effect. Hydrocortisone (cortisol) is the standard against which other GCs are compared. Cortisol, for comparative purposes, is said to have antiinflammatory and mineralocorticoid activity equal to 1 . The potency of other GCs is calculated as factors of this. Prednisone and prednisolone have intermediate anti-inflammatory activity but somewhat less mineralocorticoid activity than cortisol, while fluorinated compounds, such as dexamethasone, betamethasone, and triamicinolone have potent anti-inflammatory activity but essentially no mineralocorticoid effect (Coppoc, 1984). Binding GCs to water insoluble esters prolongs onset and duration of action by slowing absorption (Calvert and Cornelius, 1990). Glucocorticoids are released over days to weeks from moderately soluble esters and over weeks to months from poorly soluble esters such as acetonide, pivalate, and diproprionate. Esterification may also increase the antiinflammatory potency in some cases. Water soluble salts of GCs have a relatively short onset of effect. Protein binding affinity varies for different GCs. Hydrocortisone and prednisone are highly bound to transcortin and albumin. Thus, because the free drug is active, dosages should be adjusted in animals with hypoproteinemia. The biological half-life is longer than the plasma half-life (Greene, 1983). For instance, prednisolone has a plasma half-life of > 1 hour, although suppression of the HPA Axis continues for 12 to 36 hours. The effects on the immune system depend on the dosage used.

Glucocorticoids block the synthesis of prostaglandins and leukotrienes. Myeloproliferation of fibroblasts and collagen synthesis is also suppressed by GCs (Cohn, 1997). At higher doses, GCs are actually immunosuppressive.

\section{Clinicopathological changes associated their administration}

The complete blood count alterations associated with GCs therapy include neutrophilia, lymphopenia, and eosinopenia, the classic 'stress leukogram' (Cohn, 1991). In addition, GCs can increase the number of nucleated red blood cells and platelets seen in the peripheral blood (Schalm et al., 1975).

The changes in the serum chemistry panel reflect the induction of alkaline phosphatase isoenzyme production by hepatocytes in dogs. Cats have no steroid induced isoenzyme (Solter et al., 1993). Alanine transaminase is often increased moderately in dogs. Blood glucose concentration is increased to just above the normal range (Calvert and Cornelius, 1990). Serum cholesterol may be increased. Urine specific gravity is usually in the isosthenuric range. There may be mild proteinuria, especially if urinary tract infection or hypertension secondary to GCs is present. Occult urinary tract infections are common in animals on GCs, therefore, a urine sediment exam should be performed on patients on long-term therapy (Torres et al., 2005).

\section{Adverse effects of glucocorticoids}

Two categories of toxic effects result from the therapeutic use of corticosteroids: those 
resulting from withdrawal of steroid therapy and those resulting from continued use at supraphysiological doses (Goodman, 1996).

\section{Withdrawal of therapy}

The most frequent problem in steroid withdrawal is flare-up of the underlying disease for which steroids were prescribed (Goodman, 1996). The most severe complication of steroid cessation is acute adrenal insufficiency that results from overly rapid withdrawal of corticosteroids after prolonged therapy has suppressed the hypothalamic-pituitary-adrenal (HPA) axis. The exogenously administered eventually results in atrophy of the adrenal cortex that leads to hypoadrenal crisis (Greene, 1983). If appropriate treatment is not given, the animal may die.

At pharmacological doses, GCs alter the health of most cells in the body, based on their physiological effect. Besides the consequences that result from the suppression of the HPA axis, a number of other complications result from prolonged corticosteroid administration. These include fluid and electrolyte abnormalities, hypertension, hyperglycemia, increased susceptibility to infection, osteoporosis, myopathy, behavioral disturbances, cataracts, growth arrest, and the characteristic habitus of steroid overdose, including fat redistribution (Calvert and Cornelius, 1990). Although hepatic function is usually unaffected, hepatomegaly can result in abdominal discomfort and tachypnea or dyspnea (Nelson et al., 1988). Skeletal muscle weakness can result from potassium depletion and from actual loss of muscle tissue through prolonged catabolism. In some cases the muscles may stiffen because of steroid myopathy (Greene et al., 1979). Weakening of the abdominal muscles contributes to a pendulous abdomen in dogs and cats. Additionally, weakened intercostal muscles are in part responsible for the panting or tachypnea that results from steroid administration. In the gastrointestinal tract, GCs cause reduction in gastric mucus production and decreased gastric epithelial cell turnover, resulting in a tendency toward gastric ulceration (DeNovo, 1994). Reduction in the aqueous portion of pancreatic secretions and pancreatic duct cell proliferation may predispose to pancreatitis when GCs are used at high doses (Hall et al., 1988). In the case of dogs with severe spinal cord disease, their administration has been associated with fatal colonic perforation (Calvert and Cornelius, 1990). Alterations in local immune function in the small intestine may result in bacterial diarrhea. Cutaneous changes associated with long-term use of GCs include decreased cell turnover, resulting in thin skin, and an increase in bacterial infections (Calvert and Cornelius, 1990). A symmetrical loss of hair and poor regrowth of hair following clipping is also noted. As a consequence of reduced fibroblastic activity, wound healing is poor and the walls of blood vessels weaken, so promoting bruising. Calcinosis cutis, a dystrophic calcification of the dermis, may also occur in animals on chronic GCs therapy (Calvert and Cornelius, 1990). Cats are reported to be remarkably resistant to the adverse effects of exogenous corticosteroids (Scott, 1979). Because of their multiple effects to inhibit the immune system and the inflammatory response, glucocorticoid use is associated with an increased susceptibility to infection in animals. Most commonly, infections of the skin, urinary and gastrointestinal tracts are noted (Ling et al., 1979). Opportunistic systemic fungal infections can also occur. Long-term GC therapy has detrimental effects on the bones. Increased calcium resorption from the bones due to increased parathyroid hormone, inhibition of osteoblast activity, and reduced intestinal absorption of calcium can result in osteoporosis (Calvert and Cornelius, 
1990). GCs are referred to as diabetogenic and have even induced diabetes in normal animals. Thyroid hormone synthesis is reduced by GC therapy (Peterson et al., 1984). The effects of GCs on the central nervous system are varied. Polyphagia and panting are common side effects (Calvert and Cornelius, 1990). Although not as common in dogs and cats as in humans treated with GCs, mental changes can also occur. These include euphoria, hyperactivity, and depression. The long-term administration of GCs, even at low dosages, can result in all the outward signs of Cushing's disease (hyperadrenocorticism) (Feldman and Nelson, 1996). However, exogenous administration of GCs reduces $\mathrm{ACTH}$ and $\mathrm{CRH}$ secretion via negative feedback mechanisms, resulting in atrophy of the adrenal glands (Scott et al., 1982).

Ideally, most diseases that require chronic glucocorticoid administration can be maintained in remission with long-term low dose alternate-day medication.

When iatrogenic hyperadrenocorticism has been diagnosed, physiological doses (0.2 $\mathrm{mg} / \mathrm{kg}$ once daily) are required until the animal regains its own GCs production.

Five types of disease categories exist:

Physiological replacement (Calvert and Cornelius, 1990).

Management of allergic disease (Mueller and Noxon, 1990)

Immunosuppressive treatment of immunemediated disease

Adjunctive treatment in neoplastic diseases, e.g., mast cell neoplasia (McCaw et al., 1994)

As part of an antishock protocol (Haskins, 1992)

\section{Clinical summary}

When used properly, GCs can be lifesaving. However, the detrimental effects have a high price, including possible death, when they are used inappropriately. Wise selection of the type of GC and the dose in cases of diagnosed illness will usually prevent problems. Corticosteroids administered in multiple formulations (e.g., oral, parenteral, and topical) for broad ranging disorders but mainly used for their antiinflammatory and immunosuppressive effects. Except in patients receiving replacement therapy for adrenal insufficiency, glucocorticoids are neither specific nor curative, but rather are palliative. Given the number and severity of potential side effects, the decision to institute therapy with glucocorticoids always requires a careful consideration of the relative risks and benefits in each patient. After therapy is initiated, the minimal dose needed to achieve a given therapeutic effect must be determined by trial and error and must be re-evaluated periodically as the activity of the underlying disease changes or as complications of therapy arise. A single dose of glucocorticoid, even a large one, is virtually without harmful effects and a short course of therapy (up to 1 week) is unlikely to cause harm in the absence of specific contraindications. As the duration of glucocorticoid therapy increases beyond 1 week, adverse effects increase in a time- and dose-related manner. Finally, abrupt cessation of glucocorticoids after prolonged therapy is associated with the risk of adrenal insufficiency due to suppression of the HPA axis, which may be fatal. Therefore, their administration should be limited to specific conditions in which their benefits outweigh their risks.

\section{References}

Behrend, E. N. and Kemppainen, R. J. 1997. Glucocorticoid therapy: pharmacology, 
indications,

and complications. Veterinary Clinics: Small Animal Practice. 27(2): 187-213.

Bone, R. C. 1991. The pathogenesis of sepsis. Annals of Internal Medicine. 115(6): 457-469.

Boothe, D. M., Mealey, K. A.: Glucocorticoid therapy in the dog and cat, in Boothe, D. W. (ed): Small Animal Clinical Pharmacology and Therapeutics. Philadelphia, WB Saunders, 2001, Pp: 313-329.

Calvert, C. A. and Cornelius, L. M. 1990. The pharmacodynamic differences among glucocorticoid preparations. Veterinary Medicine (USA). 85: 860-865.

Cohn, L. A. 1991. The influence of corticosteroids on host defense mechanisms. Journal of Veterinary Internal Medicine. 5(2): 95-104.

Cohn, L. A. 1997. August. Glucocorticosteroids as immunosuppressive agents. In Seminars in Veterinary Medicine and Surgery (Small Animal) 12(3): 150-156.

Coppoc, G. L. 1984. Relationship of the dosage form of a corticosteroid to its therapeutic efficacy. Journal of the American Veterinary Medical Association. 185(10): 1098.

De Novo, R. 1993. Medical management of gastritis, ulcers, and erosions. In XVII Annual Waltham/OSU Symposium for the Treatment of Small Animal Diseases. USA. pp. 46-50.

Feldman, E. C. and Nelson, R.W. 1996. Hyperadrenocorticism (Cushing's syndrome). In Canine and Feline Endocrinology and Reproduction. 2nd edn. Philadelphia: Saunders. Pp: 187.

Funder, J. W. 1992. Glucocorticoid receptors. The Journal of Steroid Biochemistry and Molecular Biology: 43(5): 389-394.

Goodman, L. S. 1996. Goodman and Gilman's the pharmacological basis of therapeutics (Vol. 1549). New York: McGraw-Hill.

Greene, C. E. 1983. Pharmacology, use, and misuse of corticosteroids. In: Proceedings of the Symposium on Effective Use of Corticosteroids in Veterinary Practice. Western States Veterinary Conference: 7-19.

Greene, C. E., Lorenz, M. D., Munnell, J. F., Prasse, K. W., White, N. A. and Bowen, J. M. 1979. Myopathy associated with hyperadrenocorticism in the dog. Journal of the American Veterinary Medical Association. 174(12): 1310-1315.

Hall, J. A., Macy, D. W. and Husted, P. W. 1988. Acute canine pancreatitis. The Compendium on Continuing Education for the Practicing Veterinarian (USA).

Haskins, S.C. 1992. Management of septic shock. Journal of the American Veterinary Medical Association. 200: 1915-1924.

Ling, G. V., Stabenfeldt, G. H., Comer, K. M., Gribble, D. H. and Schechter, R. D. 1979. Canine hyperadrenocorticism: pretreatment clinical and laboratory evaluation of 117 cases. Journal of the American Veterinary Medical Association. 174(11): 1211-1215.

MacDonald, J. M. 2000. Glucocorticoid therapy, in Ettinger, S. J., Feldman, E. C. (eds): Textbook of Veterinary Internal Medicine. Philadelphia, WB Saunders, Pp: 307-317.

McCaw, D. L., Miller, M. A., Ogilvie, G. K., Withrow, S. J., Brewer Jr, W. G., Klein, M. K., Bell, F. W. and Anderson, S. K. 1994. Response of canine mast cell tumors to treatment with oral prednisone. Journal of Veterinary Internal Medicine. 8(6): 406-408.

Mueller, D. L. and Noxon, J. O. 1990. Anaphylaxis: pathophysiology and treatment. Compendium on Continuing Education for the Practicing 
Veterinarian. 12(2): 157-170.

Nelson, R. W., Feldman, E. C. and Smith, M. C. 1988. Hyperadrenocorticism in cats: Seven cases (1978-1987). Journal of the American Veterinary Medical Association. 193(2): 245-250.

Peterson, M. E., Ferguson, D. C., Kintzer, P. P. and Drucker, W. D. 1984. Effects of spontaneous hyperadrenocorticism on serum thyroid hormone concentrations in the dog. American Journal of Veterinary Research.45(10): 20342038.

Sapolsky, R. M., Romero, L. M. and Munck, A. U. 2000. How do glucocorticoids influence stress responses? Integrating permissive, suppressive, stimulatory, and preparative actions. Endocrine Reviews. 21(1): 55-89.

Schalm, O. W., Jain, N. C. and Carroll, E. J. 1975. Veterinary hematology (No. 3rd edition). Lea \& Febiger.

Scott, D. W., Kirk, R. W. and BentinckSmith, J. 1979. Some effects of shortterm methylprednisolone therapy in normal cats. The Cornell Veterinarian. 69(1): 104-115.

Scott, D. W., Manning, T. O. and Reimers, T. J. 1982. Latrogenic Cushing's syndrome in the cat. Feline Practice. 12: 30-36.

Solter, P. F., Hoffmann, W. E., Hungerford, L. L., Peterson, M. E. and Dorner, J. L. 1993. Assessment of corticosteroidinduced alkaline phosphatase isoenzyme as a screening test for hyperadrenocorticism in dogs. Journal of American Veterinary Medical Association. 203: 534-534.

Standora, J. E., Bogomolnik, A. and Slugocki, M. 2017. Steroids: History, Science, and Issues. ABC-CLIO.

Torres, S. M. F., Diaz, S. F., Nogueira, S. A., Jessen, C., Polzin, D. J., Gilbert, S. M., and Horne, K. L. 2005. Frequency of urinary tract infection among dogs with pruritic disorders receiving long-term glucocorticoid treatment. Journal of the American Veterinary Medical Association. 227(2): 239-243.

\section{How to cite this article:}

Asmita Narang and Gurpreet Singh Preet. 2019. Corticosteroids: Use or Misuse in Veterinary Medicine. Int.J.Curr.Microbiol.App.Sci. 8(08): 1183-1189.

doi: https://doi.org/10.20546/ijcmas.2019.808.139 